\title{
On the elasticity of polymer model networks containing finite loops
}

\author{
Michael Lang* \\ Leibniz Institute of Polymer Research Dresden, Hohe Stra?e 6, 01069 Dresden, Germany \\ E-mail: lang@ipfdd.de
}

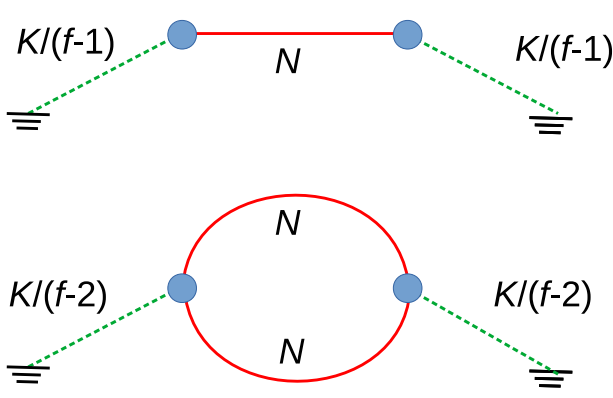

\author{
Perfect tree \\ approximation: \\ Force balance. \\ Instantaneous for- \\ mation of finite loops: \\ No force balance. \\ $\downarrow$ \\ Resistor analogy \\ is not exact
}

\begin{abstract}
Based upon the resistor analogy and using the ideal loop gas approximation (ILGA) it is shown that only pending loops reduce the modulus of an otherwise perfect network made of monodisperse strands and junctions of identical functionality. Thus, the cycle rank of the network with pending structures removed (cyclic and branched) is sufficient to characterize modulus, if the resistor analogy can be employed. It is further shown that it is impossible to incorporate finite cycles into a polymer network such that individual network strands are at equilibrium conformations while maintaining simultaneously a force balance at the junctions. Therefore, the resistor analogy provides only an approximation for the phantom modulus of networks containing finite loops. Improved approaches to phantom modulus can be constructed from considering a force balance at the junctions, which requires knowledge of the distribution of cross-link fluctuations in imperfect networks. Assuming loops with
\end{abstract}


equilibrium conformations and a force balance at all loop junctions, a lower bound estimate for the phantom modulus, $G_{\mathrm{ph}} \approx\left(\xi-c_{\mathrm{f}} L_{1}\right) k T / V$ is obtained within the ILGA for end-linked model networks and in the limit of $L_{1} \ll \xi$. Here, $L_{1}$ is the number of primary ("pending") loops, $\xi$ the cycle rank of the network, $k$ the Boltzmann constant, $V$ the volume of the sample, and $T$ the absolute temperature. $c_{\mathrm{f}}$ is a functionality dependent coefficient that is $\approx 2.56$ for junction functionality $f=3$ and $\approx 3.06$ for $f=4$, while it converges quickly towards $\approx 4.2$ in the limit of large $f$. Further corrections to phantom modulus beyond finite loops are addressed briefly.

\section{Introduction}

Recent work has revived the discussion about the elasticity of rubber networks or gels, as the detection of finite loops (also called "finite cycles") inside networks became possible from the experimental side ${ }^{1}, 2$. This advance stimulated the development of enhanced models for the elasticity of phantom networks that go beyond the ideal tree approximation ${ }^{3}-\underline{6}$. These models arrive at results that are distinct from classical work that is typically discussed in textbooks of polymer physics, which challenges our understanding of rubber elasticity.

One cornerstone of the original treatment of rubber elasticity is that phantom modulus is equivalent to the cycle rank of the network ${ }^{7}$, if cycles in pending structures are neglected. This has been argued previously using graph theory ${ }^{7}$, using micro-networks ${ }^{8}$, or within the perfect tree approximation of a perfect network where all strands are of equal degree of polymerization and all junctions of same functionality ${ }^{\underline{9}}$. Also, a numerical proof exists ${ }^{10}$ based upon the resistor analogy for polydisperse networks also within the perfect tree approximation that indicates some generality of this result.

However, real networks are made entirely of finite loops and it has been shown in recent work $^{3,4}, 11$ that these finite loops lead to a reduced contribution of the elastic strands to the phantom modulus. These results were obtained in the so-called "ideal loop gas approximation" (ILGA) where a single loop resides inside an otherwise perfect tree as a first order approximation for the impact of a finite loop on elasticity. These newer results have been challenged by a very recent paper ${ }^{5}$, 
that proposes an intermediate result in between the classical work on phantom modulus and the more recent work in Refs. $\stackrel{3,4}{.}$. It was claimed that original works ${ }^{7,10}$ are incorrect regarding finite loops that consist of $i=2$ strands. Furthermore, an additional force balance term was added to correct the resistor analogy based estimate of elasticity, that was absent in earlier work ${ }^{\underline{3}}$ or treated differently in other work ${ }^{4}$.

These conflicting results are taken in the present paper as motivation to discuss in more detail the principles for computing modulus in phantom model networks made of finite cycles. This discussion addresses several key points beginning with the resistor analogy, its limitations, force balance conditions, and the implications when generalizing the ILGA to a non-ideal network envi-

ronment. It will be shown that original works ${ }^{7,10}$ are correct when applying the resistor analogy to the ILGA. Unfortunately, this analytical result is only approximate as the resistor analogy breaks down once finite loops appear within the network since a force balance cannot be obtained within a finite loop, if the loop and the surrounding network are all made of strands at reference size. Furthermore, a lower bound for the modulus is derived for end-linked model networks under the consideration of the degree of polymerization (DP) distribution of finite loops in a network. Finally, other corrections beyond finite loops that are not considered in the classical work on phantom modulus are addressed briefly.

\section{The resistor analogy}

Let us start with the resistor analogy for the ILGA. Below, we use approach and notation of Ref. $\underline{\underline{4}}$ to double check the results of Ref. $\underline{\underline{5}}$. The basic structure that is discussed is a single loop made of $i$ strands that is incorporated into an otherwise perfect tree network. $N$ is the degree of polymerization (DP) of a network strand, $f$ the junction functionality, and $K=(f-1) N /(f-2)$ the DP that describes the virtual strand running from a junction through a single adjacent bond to the non-fluctuating elastic background. Within the resistor analog, the DP's are treated as resistors $\propto N$. Thus, $K /(f-2)$ is the $\mathrm{DP}$ (resistor) of the equivalent chain that models the net connection through the $f-2$ remaining connections to ground that run not trough the finite loop ${ }^{4}$, see Figure 1 where the simplest example of a loop of order $i=2$ is shown. 


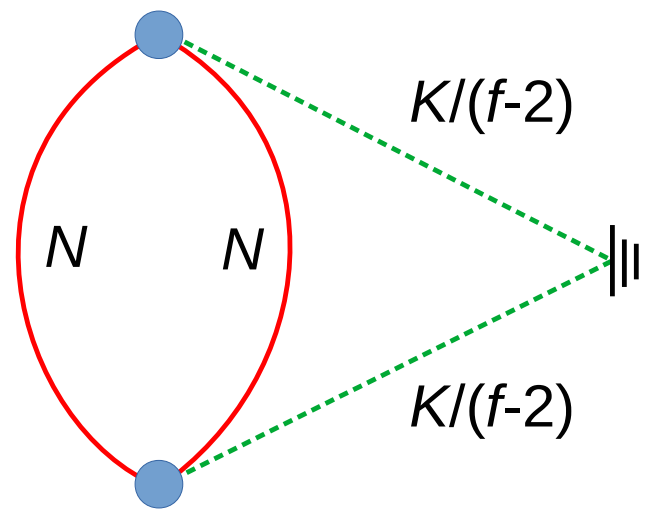

Figure 1: Resistor analog for a loop made of $i=2$ strands of $N$ segments. The blue circles represent the junctions of the loop. The virtual strands of $K /(f-2)$ describe the effective connection to ground through the $f-2$ strands of $K$ effective segments. See Ref. $\underline{\underline{4}}$ for more details.

In the resistor analogue, one asks the question: What is the portion of the current injected at two adjacent junctions (i.e. junctions that are connected directly through a single chain) that runs through a particular wire ${ }^{10}$ ? To answer this question, one removes first the "wire" under consideration from the network and computes then the conductance between the two junctions through the remaining network structure. Here, this wire is one $N$-mer of the loop $i=2$. The resulting conductance is modeled within the resistor analogy by an effective strand of $N_{\text {eff }}$ segments, where $N_{\text {eff }}$ is computed by treating it as a resistor. Thus, the total conductance between the two junctions in Figure 1 is the sum of the conductances through the remaining $N$-mer and through the two strands to ground:

$$
\frac{1}{N_{\mathrm{eff}}}=\frac{1}{N}+\frac{f-2}{2 K}
$$

The contribution of the removed $N$-mer of the loop to the total elastic strand between both junctions (after inserting it back into the loop) is then given by the ratio between the $N$-mer and the combined strand $N+N_{\text {eff }}$ as in the classical derivation of the phantom modulus 12 . One obtains here

$$
\frac{N}{N+N_{\text {eff }}}=\frac{f-2}{f}+\frac{2}{f^{2}}
$$

This contribution is larger than the ideal contribution by the last term of $2 / f^{2}$. The corresponding change in the elastic contributions of the surrounding network strands were computed in equation (S31) of the supporting information of Ref. $\underline{\underline{4}}$ providing a net reduction of the elastic contribution by 
$2 / f^{2}$, which cancels exactly the enhancement inside the loop. Therefore, there is no change in the sum of all point-to-point conductances between two adjacent junctions inside the resistor network when rewiring the strands of a perfect network to form a single loop of order $i=2$. Accordingly, the sum of all effective elastic contributions between any pair of directly connected junctions is not modified for $i=2$.

The above result is readily generalized to $i \geq 3$ with the approach presented in the supporting information of Ref. . Here, loops made of $i \geq 3$ strands are subsequently reduced to an asymmetric loop of two strands, where the corresponding strands to ground, $K_{\mathrm{av}}$ from equation (S21) of Ref. replace the $K /(f-2)$ term above and the above "bypass" of $N$ segments, (i.e. the second strand that connects the pair of junctions not through ground) is computed as $N_{\mathrm{k}}$ in equation (S16) and replaces $N$ in equation (11) above. These modifications lead consistently to an exact cancellation of the enlarged elastic contribution of the loop strands by the reduced contribution of the surrounding network strands. The supporting information contains a short FORTRAN code that performs the corresponding recursion for a range of different $i$ and $f$. The last column of the output is the net change in the point-to-point conductances of single wires between all pairs of adjacent junctions when averaged over the whole tree network with the loop in the middle. The first two columns of the output are $f$ and $i$ respectively.

The above results are identical to the discussion in Ref. $\underline{\underline{5}}$ for $i \geq 3$ and differ only for $i=2$ in the contribution of the two strands that form the loop. The difference is that above, these strands were treated independently, while these were first combined into a single strand of half the DP in Ref. $\underline{\underline{5}}$ but never split again when considering the contributions of all real network strands. Since the combination into one strand removes one elastic strand from the network, it was consistently found in Ref. $\underline{5}$ that the conductance of the structure corresponds to a network with one elastic strand removed. In this context it is worthwhile to mention that the elastic contribution of loop strands $i=2$ measured in Ref. $\underline{1}$ is smaller by a factor of two as compared to the average elastic contribution in networks made of stars with $f=4$. This observation is consistent with the simulation data presented in Figure 3 of Ref. $\underline{4}$ and the prediction for this ratio that is $(f-2) / f$ (compare equation (S29) of the supporting information of Ref. $\underline{\underline{4}}$ with the contribution of a strand within a perfect 
network). Ref. $\underline{\underline{5}}$ predicts at equation (18) that strands $i=2$ contribute only a quarter of the ideal strands, which is, therefore, neither in agreement with simulation data ${ }^{4}$ nor with experiments $\frac{1}{}$.

Altogether, there is no principal disagreement between the ILGA and older work ${ }^{7}$, where it was discussed that the cycle rank of a reduced network (where all pending structures connected to the network only through a single point have been removed) is the proper measure for the modulus of the network. This result is exact only if the resistor analogy provides an exact representation of the phantom modulus of an elastic network. However, this is not the case when finite loops appear in the network as discussed in the following section.

\section{Force balance vs. resistor analogy}

To understand why the resistor analogy breaks down for polymer networks containing finite cycles, we have to recall first that the free energy of a Gaussian strand is a function of the square ratio of the size $R$ of the strand with respect to its reference size ${ }^{12}, R_{0}=b N^{1 / 2}$. Size dependence cancels out for computing the entropy of the chain and the degree of polymerization can be interpreted as a resistance $\propto 1 / N$, when all strands are linked instantaneously with a size distribution identical to the unperturbed one at the reference state. This is one essential step for deriving, for instance, the affine model of rubber elasticity and it is the first condition that needs to be satisfied to employ the resistor analogy. Note that this condition is equivalent to considering individual elastic springs that are all stretched by thermal agitation at the instance of cross-linking and thus, are at equilibrium conformations.

The second condition is that a force balance must be achieved at each junction, since this refers to the maximum entropy of the network (in the resistor analog, the sum of all currents at a node must be zero $\left.\frac{13}{3}\right)$. Finally, we have to sum over the elastic contributions of all real strand parts of the combined chains that represent the elastic network to determine the phantom modulus correctly $\underline{\underline{6}, 14}$. This is the reason why, for instance, $N /\left(N+N_{\text {eff }}\right)$ appears in equation (2)).

To elucidate the relationship between these conditions in case of loop formation, let us consider the simplest non-pending loop $i=2$. In Figure 2, this case is simplified to the linking of two strands with $N$ segments into a loop while connecting the loop by two strands of $K /(f-2)$ 

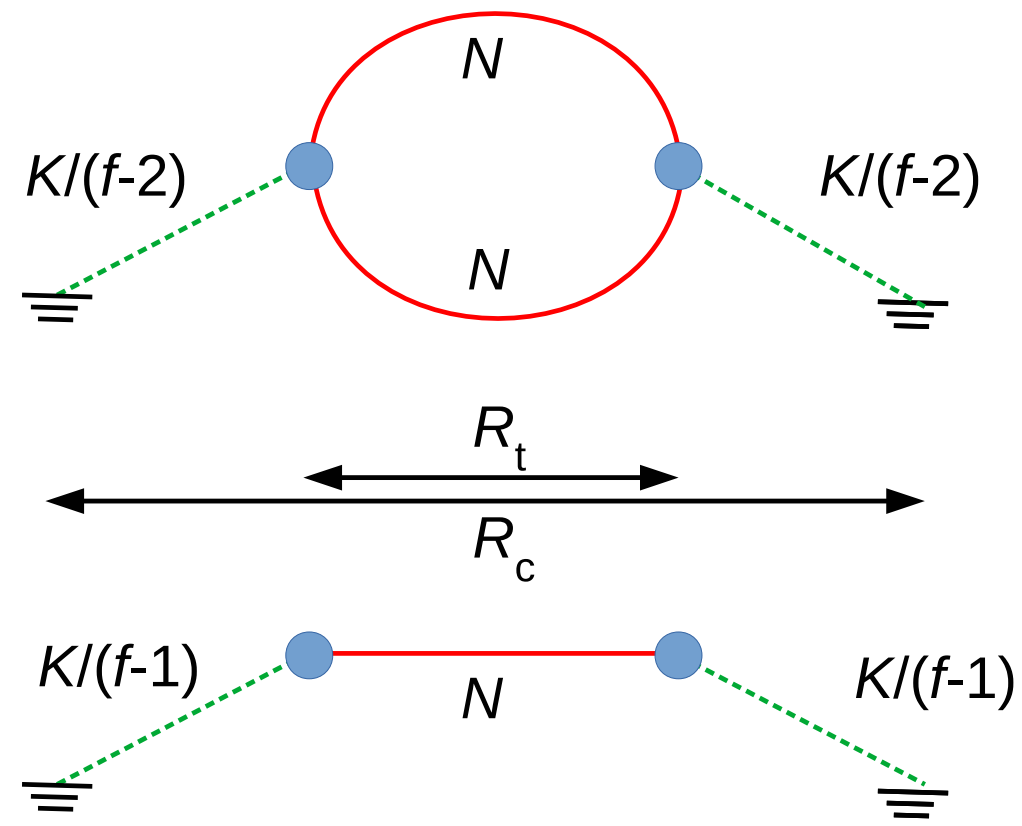

Figure 2: Comparison of a loop made of two strands, $i=2$, (top part) with a network strand in the ideal tree approximation (lower part). The red lines indicates the real strands, the green dashed lines are the virtual strands that connect to the non-fluctuating elastic background (or to ground in the resistor analogy). $R_{\mathrm{t}}$ and $R_{\mathrm{c}}$ are the time average size of the real strands and the combined strand respectively.

segments to ground. To satisfy the first condition, we have to link instantaneously all strands at their reference size. If we do so, the two "parallel" strands of the loop $i=2$ pull with twice the force against the "single" strands to ground, which does not refer to a force balance at the instance of cross-linking. But when the structure relaxes forces, the strands are no more at reference size. Thus, both conditions cannot be satisfied at a time for this "instantaneous linking" (IL) case to form finite loops.

Without loop formation as in the perfect tree approximation, there are only linear connections of single strands, see Figure2, which satisfies a force balance at the instance of cross-linking. Only in this case, the ratio between the time average square size of the real strand, $R_{\mathrm{t}}^{2}$, and the square size of the combined strand, $R_{\mathrm{c}}^{2}$, is equivalent to the portion of the combined chain that refers to the $N$-mer. Only then we can replace the ratio of these square sizes by the degree of polymerization of the real and the combined strand.

Now, let us consider that the loop is formed in reference size as characterized by a single elastic strand of $N / 2$ segments. Subsequent linking of this relaxed loop to ground results then in 
a force balance at the instance of cross-linking. Let us call this condition the "relaxed linking" (RL) condition. However, we still have to consider here that the loop contributes two real strands that are both stretched to the time average size of the loop. This latter point is essentially the difference between the results in Ref. $\underline{\underline{5}}$ for $i=2$ (where only one contribution is considered) and Refs. $\underline{3}, \underline{4}$ that consider two contributions.

The approach of Ref. $\underline{4}$ up to equation (3) and sections I-IV of the supporting information is considering RL, which assures a force balance at the instance of cross-linking such that no further relaxation of the strands must be considered. This latter point is probably not obvious, since more complex loops are mapped onto the case $i=2$ through a series of $Y-\Delta$ transforms $\frac{15}{}$. The effect of these transforms is to split the elastic forces into three disjoint contributions, that refer to a) the $N$-mer that connects both adjacent junctions directly, b) an effective second strand that connects these junctions not through ground (the "bypass"), and c) the sum of all contributions that run through ground. Bypass and direct connection are combined into a single elastic strand

$$
N_{\mathrm{X}}=\frac{N_{\mathrm{k}} N}{N_{\mathrm{k}}+N}
$$

that is linked at its reference size with the strands to ground, which refers to a simultaneous force balance at all junctions of the loop (under consideration of all loop strands and all strands to ground) for all $i \geq 2$. A very recent paper by Panyukov 11 also uses this condition to establish a force balance at the loop junctions. This procedure leads to a reduction in the elastic effectiveness within the network as compared to a perfect tree network by

$$
\Delta \epsilon_{2} \approx \frac{2(f-1)}{f^{2}}
$$

per strand of the loop for $i=2$ and by approximately

$$
\Delta \epsilon_{\mathrm{i}} \approx \frac{2(f-2)}{(f-1)^{i}}
$$

per strand of the loop for $i \geq 3$.

The opposite limit of IL was discussed in section VI of the supporting information of Ref. $\underline{\underline{4}}$. 
In comparison to RL, the elastic contributions of a strand of the loop in case of IL, $\epsilon_{\mathrm{i}, \mathrm{IL}}$, is larger than the contribution in case of RL, $\epsilon_{\mathrm{i}}$, according to

$$
\epsilon_{\mathrm{i}, I L}=\epsilon_{\mathrm{i}} \frac{N+2 K_{\mathrm{av}}}{N_{\mathrm{X}}+2 K_{\mathrm{av}}}
$$

for all $i \geq 2$, since $N_{\mathrm{X}}<N$. However, the $N_{\mathrm{X}}$ of the RL case converges quickly towards $N$ that is considered for IL, such that there is only a significant difference for the smallest $i \gtrsim 2$. Therefore, also the change in elastic effectiveness per strand of the loop converges towards equation (5) for large $i$.

Ref. $\frac{5}{}$ does not consider a force balance up to equation (25) but uses the result from the resistor analogy that each pending loops removes $k T$ of elastic energy plus an incorrect term for $i=2$ as discussed above. To remedy the missing force balance, the time average square extensions of all loop sub-strands in the network are assumed to be identical to the unperturbed loop sub-strands in sol with a corresponding reduction of size and thus, an elastic contribution $\propto 1-1 / i$. This correction refers to imposing a force balance along the loop but ignores the virtual strands to ground. These actually increase loop sizes as compared to the unperturbed size in sol, since the bypass contains $>(i-1) N$ segments for $i>2$. This is also evident from the simulation data in Figure 3 of Ref. $\stackrel{4}{ }$, where such a qualitative behavior of unperturbed loop strands can be observed indeed, however, only after dividing out the excess strain of the network strands that enlarges loop sizes as compared to isolated loops in sol. Altogether, the treatment of Ref. $\frac{5}{}$ overestimates the correction to modulus for all $i \geq 2$.

Besides the differences between IL and Rl, we have to be aware that these two limiting cases are a large simplification of the real cross-linking process. It has been discussed in a series of recent works $16-20$ that loop formation within a single polymer is a non-Markovian process that inevitably occurs at enlarged chain conformations. The discussion in these works provides strong arguments that loop formation in networks should occur also at enlarged chain conformations. Since cyclization competes with other reactions during network formation, the results of Refs. $\underline{16}-20$ are not quantitative concerning network formation. Besides these works, other arguments were put forward in recent work to explain stretched chain conformations in cross-linked networks ${ }^{21}$. 
Altogether, the assumption of equilibrium chain conformations at the instance of cross-linking has to be questioned and further investigations are necessary to understand under which conditions loops are closed and elastic chains are incorporated into the network structure.

In any of the cases mentioned above, the chains are stretched at the instance of cross-linking as compared to the chain conformations assumed in the RL case. Thus, the consideration of RL should provide a lower bound for the phantom modulus as discussed in the following section.

Finally, we have to remark that the resistor analogy can be used to compute exactly the effective elastic strand that describes the fluctuations of a given junction with respect to the position of an adjacent junction or with respect to ground. This is relevant for analyzing the relaxation dynamics of the network since the Kirchhoff matrix is the "generalized Rouse matrix" of the network ${ }^{22}$. But this connectivity based analysis is not sufficient to characterize modulus, since phantom modulus is a function of the time average the extension of the elastic network strands. A related example for this point is the superelasticity of de-swollen networks ${ }^{23}$, where the de-swelling causes shrunken chain conformations, and correspondingly, a lower phantom modulus of the network.

\section{A first estimate on the maximum impact of finite loops on}

\section{phantom modulus}

Several simulation studies addressed the average loop DP $\underline{24} \underline{25}$, the distribution of loop DP's $\underline{25-27}$, or the frequency of smallest loops $\propto N^{-1 / 2}$ in end-linked model networks 28 - 32 . The average loop DP has been estimated both analytically ${ }^{33}$ and numerically $2 \underline{25}$. Furthermore, a recursion method to compute the DP distribution of loops has been proposed ${ }^{34}$ and it was shown that the loop DP distribution scales universally $\propto i^{-5 / 2}$ at the gel point $\underline{35-38}$. For well developed end-linked model networks far beyond the gel point, there is a characteristic peak in the DP distribution ${ }^{25-27}$, that is a function of conversion $p$, junction functionality $f$, and the overlap number $P$ of elastic strands in the network ${ }^{25}$, with an additional dependence on the stoichiometric imbalance $r$ in case of networks that were established by a co-polymerization, since increasing stoichiometric imbalance drives the samples closer to the gel point. 
In comparison to these model networks, randomly cross-linked networks have a different DP distribution of smallest loops that decays $\propto N^{-3 / 2}$ with only little dependence on conversion, since pending loops result essentially from the contacts between monomers of the same polymer $\underline{39}$. While there are significant differences for the smallest loops, the peak in the DP distribution and the average loop DP agree almost quantitatively with the corresponding results for the endlinked model networks ${ }^{25}$. This latter point was used in Ref. $\underline{11}$ to estimate the characteristic loop size by considering the corresponding end-linked model networks instead of randomly cross-linked networks. But as we will see below, the smallest loops contain the least elastically effective strands. Thus, the estimate of Ref. $\stackrel{11}{\underline{1}}$ for randomly cross-linked networks is not quantitative for end-linked model networks.

For our simple first order estimate on the net impact of finite loops on phantom modulus in end-linked model networks, we make use of the result that the frequency $L_{\mathrm{i}}$ of the smallest loops made of a different number of strands, $i \neq j$, can be related $\frac{31,37}{1 h r o u g h}$

$$
\frac{L_{\mathrm{i}}}{L_{\mathrm{j}}} \approx[p(f-1)]^{|i-j|}(j / i)^{5 / 2}
$$

This relation is obtained from adopting a mean-field estimate for the average number of $\approx$ $f[p(f-1)]^{i-1}$ connected units in generation $i$ away within an incomplete Bethe lattice where only a portion $p$ of bonds is existing. The number of connected units provides the expected number of attempts to close a cycle containing $i$ strands, while the probability for closure is related to the return probability $\propto(i N)^{-3 / 2}$ of a random walk containing $i$ strands. The power $5 / 2$ results from integrating this return probability up to conversion $p$, see the discussion following equation (20) in Ref. .37 .

Equation (7) can be applied as long as the cycle rank, $\xi$, is large compared to the frequency of the smallest loops, $\xi \gg R_{1}$, since then, the formation of finite loops is only a weak distortion of the incomplete Bethe lattice at small $i$. Note that the above relation refers to the simplest case of a homo-polymerization of $f$ functional star molecules and is based upon the assumption that adjacent loops are formed independently from each other. More complex architectures including end-linked model networks are discussed elsewhere $\underline{37,40}$. 
Recall that in equation (5) , the impact per loop strand is $\propto(f-1)^{-i}$ for $i \geq 3$. With this dependence, we can relate the net effect of loops with a different number of elastic strands $i>3$ in well developed networks within the above approximations:

$$
\frac{i}{3} \frac{\Delta \epsilon_{\mathrm{i}} L_{\mathrm{i}}}{\Delta \epsilon_{3} L_{3}} \approx p^{i-3}\left(\frac{3}{i}\right)^{3 / 2} .
$$

Here, the numerical coefficient of $i / 3$ reflects that all $i$ strands of a loop reduce modulus by $\Delta \epsilon_{i}$. Thus, the net impact of loops $i \geq 3$ can be written as

$$
\sum_{i=3}^{\infty} i \Delta \epsilon_{\mathrm{i}} L_{\mathrm{i}} \approx 3^{5 / 2} \Delta \epsilon_{3} L_{3} \sum_{i=3}^{\infty} p^{i-3} i^{-3 / 2} .
$$

The quick decay as a function of $i$ in this equation shows that still the largest net corrections to the ideal phantom modulus result from the smallest $i$ despite the fact that much more strands are part of large loops.

Simulation data on the smallest $\operatorname{loops}^{25}$ that can be found for a given strand with a spanning tree approach ${ }^{41}$ indicate that there is a characteristic loop DP of $i_{c} N$ (a typical example were $i_{c} \approx 8$ for $N \approx 20$ and $f=4$ at $p \approx 1$, see Ref. ${ }^{25}$ ) at which the distribution of smallest loops obtains a maximum. However, the strands that constitute such small loops are also part of a huge number of larger loops that can be identified within the network. We take equation (7) as a mean-field estimate for the abundance of these larger loops and thus, we do not truncate the summation at the characteristic loop DP. Instead, we use the infinite sum for a simplified mean-field estimate for the net impact of all of these larger loops. Note that in the case of full conversion, $p=1$, the infinite sum is at its maximum but remains a number of order unity: $\sum_{i=3}^{\infty} i^{-3 / 2} \approx 1.2588 \ldots \approx 5 / 4$.

Altogether, we expect that the modulus $G$ close to full conversion, $p \approx 1$, is smaller than the ideal phantom modulus

$$
G_{\mathrm{id}}=\xi k T / V=\frac{f-2}{f} \nu k T
$$

where $\nu$ is the density of network strands, $k$ the Boltzmann constant, $T$ the absolute temperature, and $V$ the volume of the sample. A lower bound for modulus is estimated by considering the 
corrections due to finite loops in the RL case

$$
\begin{gathered}
G \approx G_{\mathrm{id}}\left\{1-\frac{L_{1}}{\xi}-2 \Delta \epsilon_{2} \frac{L_{2}}{\xi}-\sum_{i=3}^{\infty} i \Delta \epsilon_{\mathrm{i}} \frac{L_{i}}{\xi}\right\} \\
\approx G_{\mathrm{id}}\left\{1-\frac{L_{1}}{\xi}\left(1+2^{-3 / 2}(f-1) \Delta \epsilon_{2}+\frac{5}{4}(f-1)^{2} \Delta \epsilon_{3}\right)\right\} \\
\approx G_{\mathrm{id}}\left\{1-\frac{L_{1}}{\xi}\left[1+\frac{(f-1)^{2}}{\sqrt{2} f^{2}}+\frac{5(f-2)}{2(f-1)}\right]\right\} \\
=\frac{k T}{V}\left(\xi-c_{\mathrm{f}} L_{1}\right) .
\end{gathered}
$$

Above we introduced $c_{\mathrm{f}}$ as short hand notation for the term in the square brackets. Note that $c_{\mathrm{f}}=7 / 2+2^{-1 / 2}$ for $f \rightarrow \infty$. For $f=3$, there is $c_{\mathrm{f}} \approx 2.56$ and for $f=4$, there is $c_{\mathrm{f}} \approx 3.06$. Thus, the net correction to modulus due to loops with $i \geq 2$ is larger than the correction due to pending loops $i=1$ for all $f \geq 3$.

In practice, $L_{1}$ or $L_{2}$ can be determined through one of the available methods ${ }^{1,2}$ or by MALDITOF experiments $\frac{42}{2}$ on an analog $f=2$ system, while $\xi$ can be computed from the conversion of the reactive groups in the gel $\underline{43,44}$. As pointed out in previous work ${ }^{37}, \underline{40,45}$, we have to emphasize that cyclization is a unique function of the overlap number $P \propto \phi N^{1 / 2}$ and the junction functionality. Thus, at the absence of a quantitative method for counting loops, one can still analyze qualitatively the corrections to modulus as a function of the overlap number by recalling that $\underline{37}$

$$
L_{1} \propto \frac{f-1}{\phi N^{1 / 2}}
$$

for the practically important limit of $L_{1} \ll \xi$ and small $f$ where multiple pending loops per junction can be neglected and where the impact of finite loops is just a correction to the phantom modulus.

For a quantitative test of our discussion, we compare equation (11) with the data of Refs. . $^{3}$. We assume $p=1$ as the effect of incomplete conversion is discussed in $\operatorname{Ref}^{\underline{5}}$ but no information on conversion is presented that would allow to shift the experimental data accordingly. For infinite stoichiometric end-linked model networks at $p=1$, there are $f / 2-1$ loops per network junction (ignoring loops in the sol). Thus, the number of primary loops per junction, $x_{1}$, of Ref $\frac{5}{5}$ is related 
to the fraction of primary loops among all loops through $L_{1} / \xi=2 x_{1} /(f-2)$. Thus, we expect that the experimental data should be above

$$
G_{\mathrm{id}}\left(1-\frac{c_{\mathrm{f}} L_{1}}{\xi}\right) \approx\left(f-2-2 c_{\mathrm{f}} x_{1}\right) \nu k T / f
$$

and below the pending loop correction (which is the estimate resulting from the resistor analogy) with $c_{\mathrm{f}}=1$. Indeed these limits set apparently an upper and a lower bound to the experimental data of Refs. $\stackrel{3}{\underline{3}}$, see Figure 3 .

In Ref. $\underline{\underline{5}}$, the gel point loop DP distribution $\propto i^{-5 / 2}$ was used in contrast to equation (7), which largely underestimates the frequency of loops $i \geq 2$, while the impact of finite loops was overestimated for $i \geq 2$ as discussed in the preceding section. The resulting agreement with experimental data in Ref. $\underline{5}$ is, therefore, less the indication of a precise approximation than merely the fortuitous cancellation of the following corrections that we have not yet taken into account.

First, the above treatment is based upon the impact of a single loop in an otherwise perfect network, which is a rather rough approximation, since essentially the whole network consists of loops of similar DP as the one that is considered. In a network of loops, the junction fluctuations will be further enhanced as the junctions are connected to less neighboring junctions in the further environment. Larger junction fluctuations imply a smaller elastic contribution as the real part of the corresponding combined strands is being reduced.

Second, with increasing conversion, it is expected that reactions involve junctions that are increasingly apart from each other. This holds in particular when the available number of reactive groups drops below one per pervaded volume of a network strand. Entanglements provide a bias towards enlarged chain conformations at cross-linking as argued in Ref. $\underline{\underline{21}}$ and loop closure in general occurs at enlarged chain conformations $\underline{\underline{16}-\underline{20}}$. Furthermore, the IL case discussed above occurs at enlarged chain conformations as compared to the RL limit. As all these cases lead to larger contributions to modulus as the estimate in equation [13, the latter must be a lower limit for modulus, given that all other corrections (besides enlarged chain conformations at loop closure) were treated correctly.

Third, the contribution of entanglements to modulus is ignored in the above discussion. This 


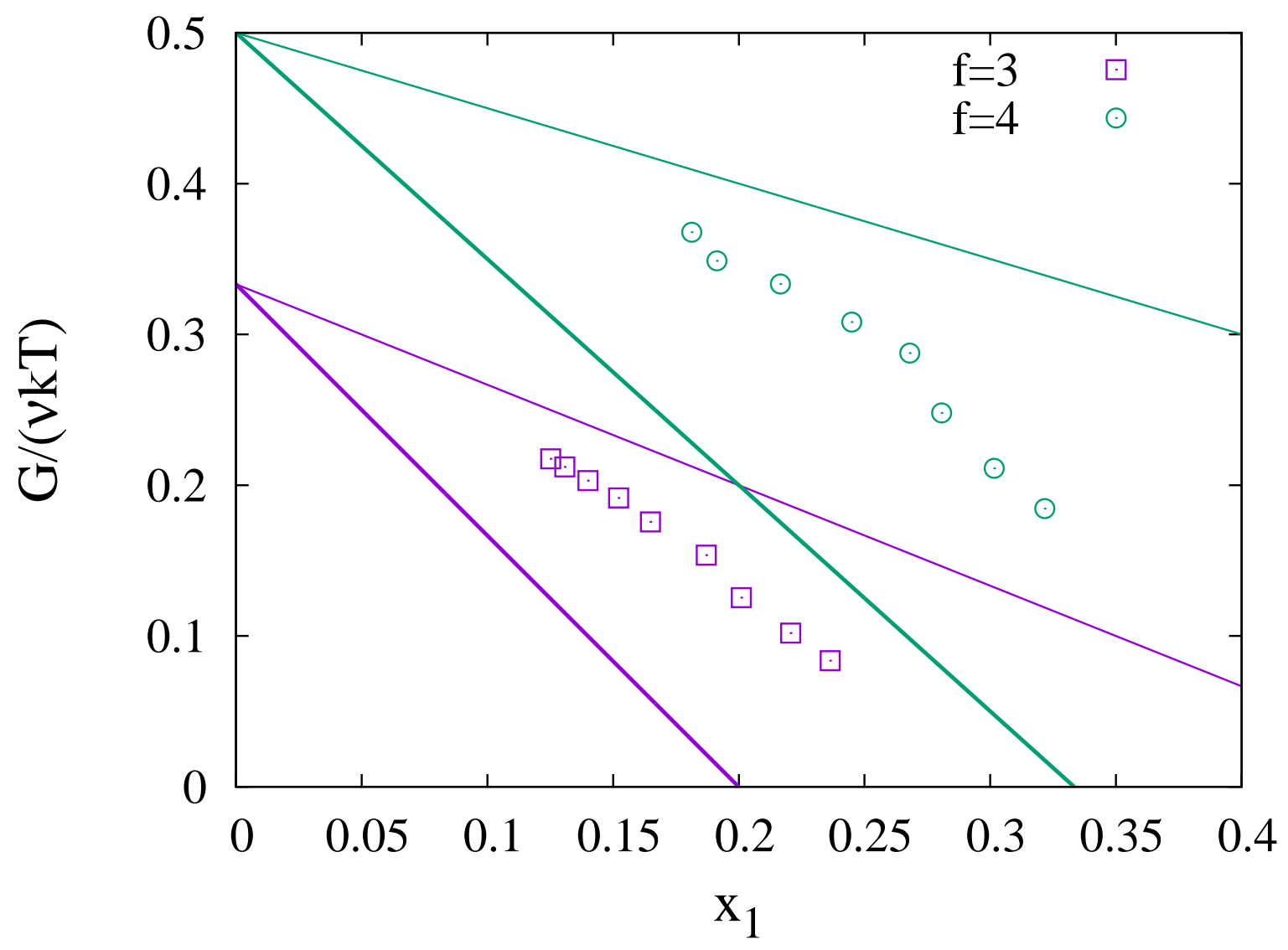

Figure 3: Comparison of the loop dependence of modulus data of Ref. $\cdot \underline{5}$ with the lower bound of equation (13) as shown by the thick lines, while the thin lines indicate the corrections due to pending loops only, $c_{\mathrm{f}}=1$, which is equivalent to the estimate from the resistor analogy. 
contribution is a function of the weight fraction of elastically active polymer at cross-linking conditions, $\omega$, and scales $\propto \omega^{7 / 3}$ as discussed in Refs. $\stackrel{12,46}{ }$. This contribution cannot be ignored by arguing that the precursor polymers were not entangled, since the active network strands are parts of "infinitely long chains" that are always above a finite concentration-dependent entanglement degree of polymerization. Since the data in Ref. $\frac{3}{3}$ was obtained by diluting the reaction mixture, there is an additional small entanglement contribution to modulus hidden in Figure 3 , which scales roughly $\propto x_{1}^{-7 / 3}$, if $\omega \approx \phi$ and $x_{1} \sim \phi^{-1}$.

Finally, the original analysis of primary loops indicated a significant amount of pending chains, see Figure 4c of Ref. $\stackrel{2}{2}$. In subsequent work by the same group ${ }^{3}, \underline{5}$, the corresponding conversion $p<1$ was not provided. The measured modulus, therefore, must lie below the ideal prediction, which is not yet accounted for in the analysis.

Altogether, the ILGA is probably a reasonable first step towards the development of more advanced models for phantom modulus. But it is not the only correction that is relevant for an improved estimate of the phantom modulus. One key problem for further development of theory is a self-consistent representation of the surrounding network structure, which is addressed to some extent in the following section.

\section{Some remarks on imperfect and polydisperse networks}

One of the largest simplifications in the computations of Refs. $\underline{3}^{-5}$ is that the surrounding network is represented by the very same elastic strand to ground at any junction. In real networks, there are pending chains, loops of different $i$, polydisperse strands, etc ... that may cause a broad distribution of elastic strands to ground and it is not a priori clear that this distribution can be represented by a single (average) effective strand. Therefore, we consider in the present section the effect of a distribution of elastic strands on elasticity and junction fluctuations.

As the simplest possible example, let us consider a perfect tree network made of $f$-functional junctions that are connected by a polydisperse distribution of chains that is described by a number fraction distribution $n_{\mathrm{N}}$. It has been shown previously ${ }^{8}, 10$, that one recovers the classical phantom coefficient of $(f-2) / f$ also in the case of polydisperse networks within the resistor analogy and the 
ideal tree approximation. The author of the present work has repeated the numerical calculations of Ref. 10 for several model distributions and can confirm that this result is correct independent of the shape of the distribution. But as shown for the numerical example below, average quantities (except of modulus) like junction fluctuations, elastic strands to ground, or network strands between junctions are not representative of the behavior of the full distributions.

As a representative numerical example, let us consider a most probable distribution of elastic strands between the junctions that is characterized by a number fraction distribution of

$$
n_{\mathrm{N}}(p)=(1-p) p^{N-1}
$$

where $p$ is the probability that a monomer is not cross-linked. The corresponding number average degree of polymerization is $N_{\mathrm{n}}=(1-p)^{-1}$ and the weight fractions of $N$-mers are here

$$
w_{\mathrm{N}}(p)=N(1-p)^{2} p^{N-1}
$$

Recall from equation (11) that conductances are required to compute the elastic contribution of network strands. Equation (14) leads for this model case to an ensemble average "conductance", $1 / \bar{N}$ of the polymers between two junctions, for which a simple analytical expression is available

$$
\frac{1}{\bar{N}}=(1-p) \sum_{N>0} \frac{p^{N-1}}{N}=\frac{(1-p) \ln (1-p)}{-p}=\frac{\ln \left(N_{\mathrm{n}}\right)}{N_{\mathrm{n}}-1}
$$

Note that $1 / \bar{N}$ is not $\propto N_{\mathrm{n}}^{-1}$ as the phantom modulus. Similarly, one finds with the self-consistent numerical approach introduced in Ref. 10 that also the average conductance to ground, $1 / \bar{K}$, is not a simple function of neither $1 / \bar{N}$ nor $N_{\mathrm{n}}^{-1}$. This is illustrated in Figure 4 that shows several ratios between $\bar{K}, \bar{N}$, and $N_{\mathrm{n}}$, which all are not constant as a function of the cross-link density. Therefore, the average $\bar{K}$ and $\bar{N}$ do not compensate each other for producing a constant phantom coefficient of $(f-2) / f$. Compensation is only achieved when the full distributions for $\bar{K}$ and $\bar{N}$ are taken into account.

The reason for this point is that the distributions of $K$ drift and change shape as a function 


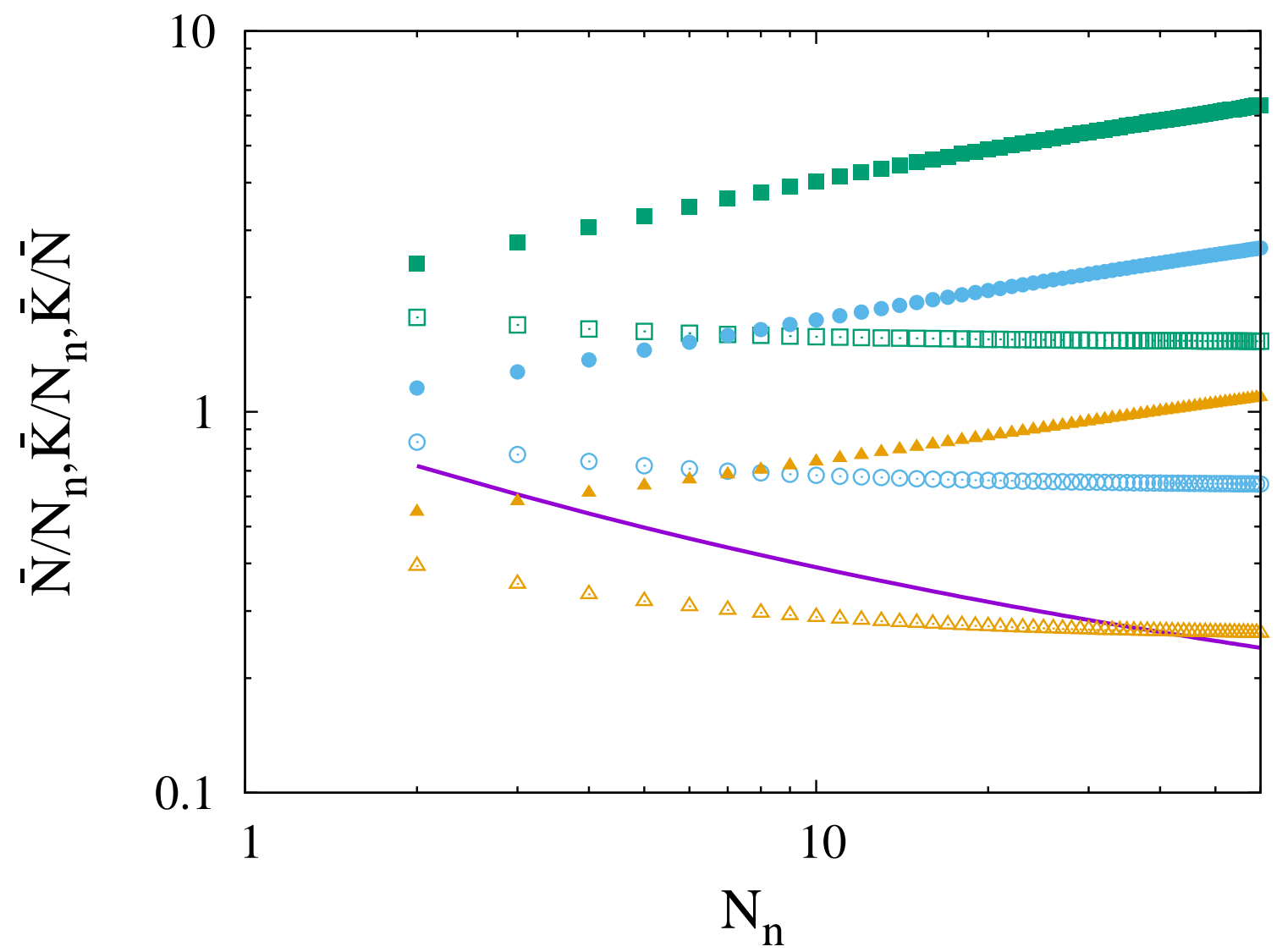

Figure 4: Comparison of average conductances $1 / \bar{N}$, the number average degree of polymerization $N_{\mathrm{n}}$ and the average conductance to ground, $1 / \bar{K}$ for perfect ideal tree network with a most probable weight distribution of strands. The line is $\bar{N} / N_{\mathrm{n}}$, the squares, circles, and triangles refer to $f=3$, 4, 6 respectively. Open symbols are $\bar{K} / N_{\mathrm{n}}$, while closed symbols refer to $\bar{K} / \bar{N}$. 


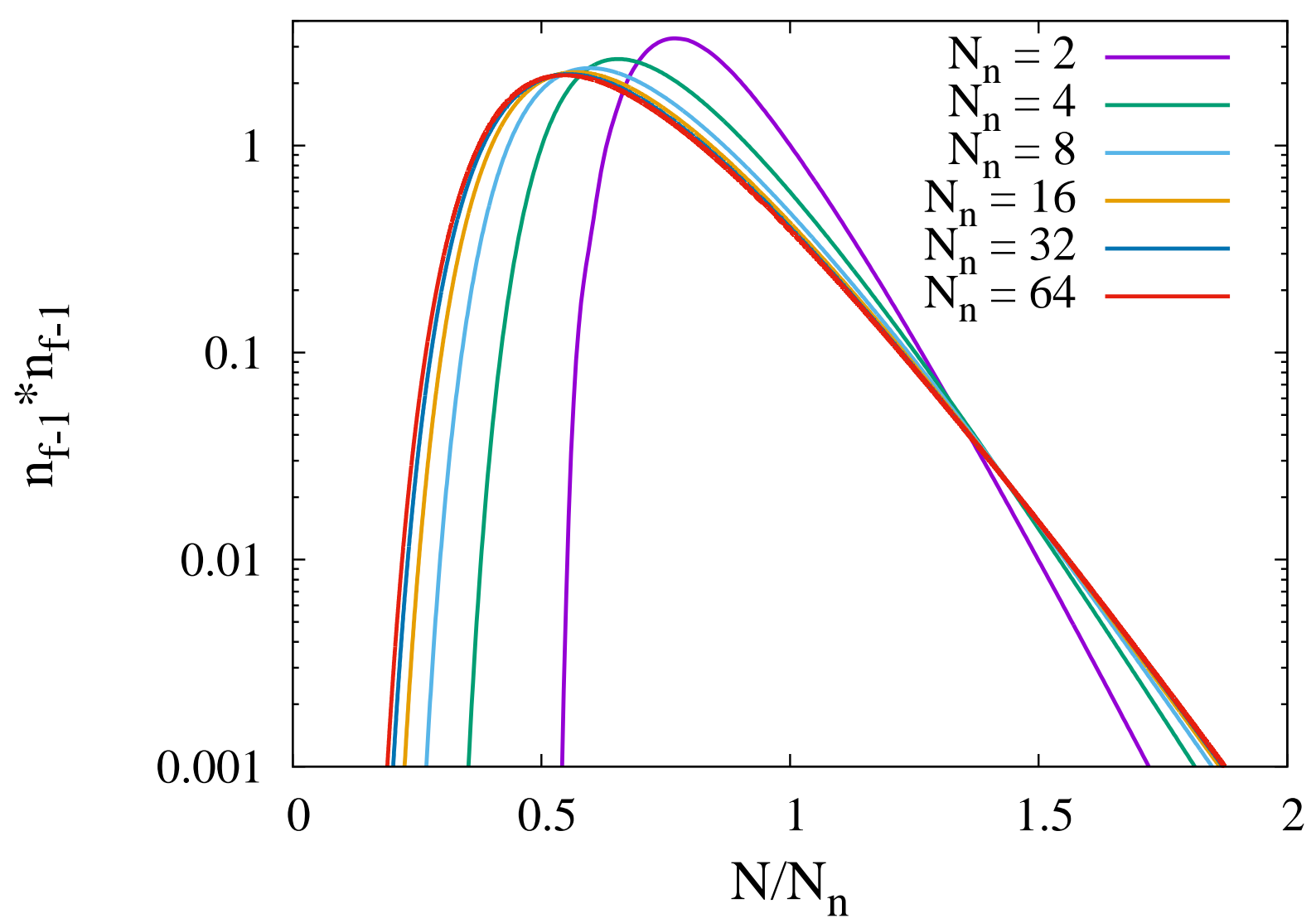

Figure 5: Distribution of the virtual strands to ground, $n_{\mathrm{f}-1} * n_{\mathrm{f}-1}$, for different $N_{\mathrm{n}}$. 
of $N_{\mathrm{n}}$. In consequence, also the net distribution $n_{\mathrm{f}-1}$ of the $f-1$ virtual strands to ground at one junction changes shape as a function of $N_{\mathrm{n}}$. This is shown in Figure 5 for the convolution of the virtual strand distributions at both junctions, $n_{\mathrm{f}-1} * n_{\mathrm{f}-1}$. Relevant for elasticity is the distribution of the combined strands $n_{\mathrm{c}}$ that is given through yet another convolution with the distribution of network strands, $n_{\mathrm{N}}$ :

$$
n_{\mathrm{c}}=n_{\mathrm{f}-1} * n_{\mathrm{f}-1} * n_{\mathrm{N}},
$$

Note that in Refs. $\stackrel{47,48}{ }$ it was discussed that the ensemble average vector order parameter in a model network is the inverse of the degree of polymerization of the corresponding combined chain. Thus, we expect that the distribution of the inverse $n_{\mathrm{c}}$ values should be relevant to characterize a polydisperse or imperfect network.

Let us denote by $n_{\mathrm{c}}(N)$ the distribution of combined strands that is obtained for a given $N$-mer in the middle instead of considering the full distribution $n_{\mathrm{N}}$. With this notation and the results of preceding work ${ }^{8,10}$, we can write for the coefficient of the phantom model of a polydisperse perfect tree network that

$$
\begin{gathered}
\frac{G_{\mathrm{ph}}}{\nu k T}=\sum_{N=1}^{\infty} n_{\mathrm{N}} \sum_{n_{\mathrm{c}}(N)} \frac{N}{n_{\mathrm{c}}(N)} \\
=N_{\mathrm{n}} \sum_{N=1}^{\infty} w_{\mathrm{N}} \sum_{n_{\mathrm{c}}(N)} \frac{1}{n_{\mathrm{c}}(N)}=\langle m\rangle N_{\mathrm{n}}=\frac{f-2}{f} .
\end{gathered}
$$

Here, $\langle m\rangle$ is the ensemble average vector order parameter (the ensemble average residual bond

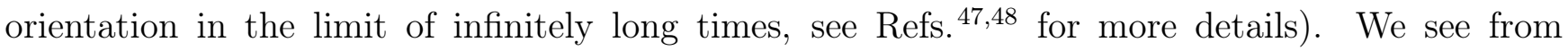
equation (18) that the computation of the ensemble average elastic contribution of the network strands refers to a weight average where the real chain part of the combined elastic strands serves as a weight for the inverse distribution of combined strands, $n_{\mathrm{c}}(N)^{-1}$. The first expression in second line of equation (18) (without $N_{\mathrm{n}}$ ) is the summation rule for taking the ensemble average residual bond orientation. Thus, the multi quantum nuclear magnetic resonance (MQ-NMR) as described in Refs. $\underline{49}, 50$ is the method of choice to detect the distribution of elastic strands in networks where the phantom model provides a reasonable approximation for the shear modulus of the network. Further development of the theory of phantom modulus can use this connection for a test of model predictions against experimental data. 


\section{Summary}

It was shown using the resistor analogy that only pending cycles within an otherwise "perfect tree" network of monodisperse strands affect the average elastic strand between adjacent junctions in the network. Therefore, one arrives within the resistor analogy at the result that the modulus of such idealized model networks should be fully determined by the cycle rank after removing pending cycles $^{\underline{\underline{T}}}$. It was further confirmed numerically that polydispersity has no effect on elasticity, if the resistor analogy can be applied in agreement with earlier work $\stackrel{8}{\underline{8} 10}$. Both elasticity and the residual bond orientation as accessible through NMR, result from a weight average of the inverse of the distribution of combined elastic strands (containing the real polymer and the virtual strands to ground). Thus, NMR is the method of choice to analyze the distribution of elastic strands, in particular in imperfect or poly-disperse networks. This is of interest also for networks made of finite loops, as the most important non-pending finite loop $i=2$ can be separated for appropriate parameters of the experiments $\stackrel{1}{1}$.

The resistor analogy is no more exact once finite loops appear in a network, since it is not possible to link the strands of the loop at equilibrium conformations (of individual strands) while maintaining simultaneously a force balance at the junctions. Assuming loops with equilibrium conformations and a force balance at all loop junctions, a lower bound estimate for the phantom modulus, $G_{\mathrm{ph}} \approx\left(\xi-c_{\mathrm{f}} L_{1}\right) k T / V$ is obtained, where $L_{1}$ is the number of primary ("pending") loops among the number of independent loops (the cycle rank $\xi$ ) of the network, $k$ the Boltzmann constant, and $T$ the absolute temperature. $c_{\mathrm{f}}$ is a functionality dependent coefficient that is $\approx 2.56$ for $f=3$ and $\approx 3.06$ for $f=4$, while it converges quickly towards $\approx 4.2$ in the limit of large $f$. The resistor analogy provides an upper bound for modulus with $c_{\mathrm{f}}=1$ that reflects the impact of pending loops only.

Further model development is necessary to understand loop closure inside a network and the corresponding enlarged loop conformations. Furthermore, a realistic self-consistent model for the surrounding network made of finite cycles, containing defects, and built of polydisperse strands must be developed. The recent paper by Panyukov $\underline{11}$ is a big step forward in this direction. The discussion in the present work provides some guidelines for the construction of a successful model. 


\section{Associated Content}

Supporting Information The supporting information (PDF) contains a brief FORTRAN code that provides a numerical proof for the resistor analogy within the ideal loop gas approximation (ILGA).

\section{Acknowledgements}

This work was supported by the Deutsche Forschungsgemeinschaft (DFG) throgh Grants LA2735/51 and LA2735/6-1.

\section{References}

(1) Lange, F.; Schwenke, K.; Kurakazu, M.; Akagi, Y.; Chung, U. I.; Lang, M.; Sommer, J.U.; Sakai, T.; Saalwächter, K. Connectivity and structural defects in model hydrogels: A combined proton NMR and Monte Carlo simulation study. Macromolecules 2011, 44, 96669674.

(2) Zhou, H.; Woo, J.; Cok, A. M.; Wang, M.; Olsen, B. D.; Johnson, J. A. Counting primary loops in polymer gels. PNAS 2012, 109, 19119-19124.

(3) Zhong, M.; Wang, R.; Kawamoto, K.; Olsen, B. D.; Johnson, J. A. Quantifying the impact of molecular defects on polymer network elasticity. Science 2016, 353, 1264-1268.

(4) Lang, M. On the elasticity of phantom model networks with cyclic defects. ACS Macro Letters 2018, 7, 536-539.

(5) Lin, T.-S.; Wang, R.; Johnson, J. A.; Olsen, B. D. Revisiting the Elasticity Theory for Real Gaussian Phantom Networks. Macromolecules 2019, 52, 1685-1694.

(6) Gusev, A. A. Numerical Estimates of the Topological Effects in the Elasticity of Gaussian Polymer Networks and Their Exact Theoretical Description. Macromolecules 2019, 52, 32443251. 
(7) Flory, P. J. Elastic activity of imperfect networks. Macromolecules 1982, 15, 99-100.

(8) Graessley, W. W. Elasticity and Chain Dimensions in Gaussian Networks. Macromolecules $\mathbf{1 9 7 5}, 8,865-868$.

(9) Flory, P.; Gordon, M.; McCrum, N. Statistical thermodynamics of random networks. Proceedings of the Royal Society of London. Series A, Mathematical and Physical Sciences 1976, $351,351-380$.

(10) Higgs, P.; Ball, R. Polydisperse polymer networks : elasticity, orientational properties, and small angle neutron scattering. Journal de Physique 1988, 49, 1785-1811.

(11) Panyukov, S. P. Loops in polymer networks. Macromolecules 2019, 52, 4145-4153.

(12) Rubinstein, M.; Colby, R. H. Polymer Physics; Oxford University Press, 2003.

(13) De Gennes, P. G. On a relation between percolation theory and the elasticity of gels. Journal de Physique Lettres 1976, 37, L1-L2.

(14) James, H. M.; Guth, E. Simple Presentation of Network Theory of Rubber, with a Discussion of Other Theories. J. Polym. Sci. 1949, 4, 153-182.

(15) Kenelly, A. E. Equivalence of triangles and three-pointed stars in conducting networks. Electrical World and Engineer 1899, 34, 413-414.

(16) Guerin, T.; Benichou, O.; Voituriez, R. Non-Markovian polymer reaction kinetics. Nature Chemistry 2012, 4, 568-573.

(17) Guerin, T.; Benichou, O.; Voituriez, R. Reactive conformations and non-Markovian cyclization kinetics of a Rouse polymer. J. Chem. Phys. 2013, 138, 094908.

(18) Guerin, T.; Dolgushev, M.; Benichou, O.; Voituriez, R.; Blumen, A. Cyclization kinetics of Gaussian semiflexible polymer chains. Phys. Rev. E 2014, 90, 052601. 
(19) Levernier, N.; Dolgushev, M.; Benichou, O.; Blumen, A.; Guerin, T.; Voituriez, R. NonMarkovian closure kinetics of flexible polymers with hydrodynamic interactions. J. Chem. Phys. 2015, 143, 204108.

(20) Dolgushev, M.; Guerin, T.; Blumen, A.; Benichou, O.; Voituriez, R. Contact Kinetics in Fracatal Macromolecules. Phys. Rev. Lett 2015, 115, 208301.

(21) Lang, M. Relation between Cross-Link Fluctuations and Elasticity in Entangled Polymer Networks. Macromolecules 2017, 50, 2547-2555.

(22) Gurtovenko, A. A.; Blumen, A. Generalized Gaussian Structures: Models for Polymer Systems with Complex Topologies. Adv. Polym. Sci. 2005, 182, 171-282.

(23) Obukhov, S. P.; Rubinstein, M.; Colby, R. H. Network Modulus and Superelasticity. Macromolecules 1994, 27, 3191-3198.

(24) Michalke, W.; Lang, M.; Kreitmeier, S.; Göritz, D. Simulations on the number of entanglements of a polymer network using knot theory. Phys. Rev. E 2001, 64, 012801.

(25) Lang, M.; Kreitmeier, S.; Göritz, D. Trapped Entanglements in Polymer Networks. Rubber Chemistry and Technology 2007, 80, 873-894.

(26) Schulz, M.; Sommer, J.-U. Monte Carlo studies of polymer network formation. The Journal of Chemical Physics 1992, 96, 7102-7107.

(27) Michalke, W.; Kreitmeier, S.; Göritz, D. Monte Carlo studies of polymer network formation. The Journal of Chemical Physics 2002, 117, 6300-6307.

(28) Leung, Y. K.; Eichinger, B. E. Computer simulation of end-linked elastomers. I. Trifunctional networks cured in the bulk. J. Chem. Phys. 1984, 80, 3877-3884.

(29) Grest, G. S.; Kremer, K.; Duering, E. R. Kinetics of end crosslinking in dense polymer melts. Europhys. Lett. 1992, 19, 195-200.

(30) Gilra, N.; Cohen, C.; Panagiotopoulos, A. Z. A Monte Carlo study of the structural properties of end-linked polymer networks. J. Chem. Phys. 2000, 112, 6910-6916. 
(31) Schwenke, K.; Lang, J.-U., M. Sommer On the Structure of Star-Polymer Networks. Macromolecules 2011, 44, 9464-9472.

(32) Lang, M.; Schwenke, K.; Sommer, J.-U. Short cyclic structures in polymer model networks: A test of mean field approximation by monte carlo simulations. Macromolecules 2012, 45, 4886-4895.

(33) Cai, L. H.; Panyukov, S.; Rubinstein, M. Hopping diffusion of nanoparticles in polymer matrices. Macromolecules 2015, 48, 847-862.

(34) Lang, M.; Michalke, W.; Kreitmeier, S. A statistical model for the length distribution of meshes in a polymer network. The Journal of Chemical Physics 2001, 114, 7627-7632.

(35) Kilb, R. W. Dilute Gelling Systems. I. The Effect of Ring Formation on Gelation. The Journal of Chemical Physics 1958, 62, 969-971.

(36) Suematsu, K. Theory of gel point in real polymer solutions. European Physical Journal B 1998, 6, 93-100.

(37) Lang, M.; Göritz, D.; Kreitmeier, S. Intramolecular reactions in randomly end-linked polymer networks and linear (Co)polymerizations. Macromolecules 2005, 38, 2515-2523.

(38) Wang, R.; Lin, T.-S.; Johnson, J. A.; Olsen, B. D. Kinetic Monte Carlo Simulation for Quantification of the Gel Point of Polymer Networks. ACS Macro Letters 2017, 4, 14141419.

(39) Lang, M.; Göritz, D.; Kreitmeier, S. Length of subchains and chain ends in cross-linked polymer networks. Macromolecules 2003, 36, 4646-4658.

(40) Suematsu, K. Recent Progress in Gel Theory: Ring, Excluded Volume, and Dimension. Advances in Polymer Science 2002, 156, 137-214.

(41) Lang, M.; Michalke, W.; S., K. Optimized Decomposition of Simulated Polymer Networks Into Meshes. Macromolecular Theory and Simulations 2001, 10, 204-208. 
(42) Kricheldorf, H. R.; Böhme, S.; Schwarz, G. Macrocycles. 17. The role of cyclization in kinetically controlled polycondensations. 2. Polyamides. Macromolecules 2001, 34, 8879-8885.

(43) Miller, D. R.; Macosko, C. W. A New Derivation of Post Gel Properties of Network Polymers. Macromolecules 1976, 9, 206-211.

(44) Lang, M. Bildung und Struktur von polymeren Netzwerken, Dissertation. Ph.D. thesis, Universität Regensburg, 2004.

(45) Wang, R.; Alexander-Katz, A.; Johnson, J. A.; Olsen, B. D. Universal Cyclic Topology in Polymer Networks. Physical Review Letters 2016, 116, 188302.

(46) Adam, M.; Delsanti, M. Viscosity and longest relaxation time of semi-dilute polymer solutions. I. Good solvent. Journal de Physique 1983, 44, 1185-1193.

(47) Lang, M.; Sommer, J.-U. Analysis of Entanglement Length and Segmental Order Parameter in Polymer Networks. Physical Review Letters 2010, 104, 177801.

(48) Lang, M. Monomer fluctuations and the distribution of residual bond orientations in polymer networks. Macromolecules 2013, 46, 9782-9797.

(49) Cohen-Addad, J. P. Effect of the anisotropic chain motion in molten polymers: the solidlike contribution of the nonzero average dipolar coupling to NMR signals theoretical description. J. Chem. Phys. 1973, 60, 2440-2453.

(50) Saalwächter, K. Proton multiple-quantum NMR for the study of chain dynamics and structural constraints in polymeric soft materials. Prog. Nucl. Magn. Res. Spectr. 2007, 51, 1-35. 

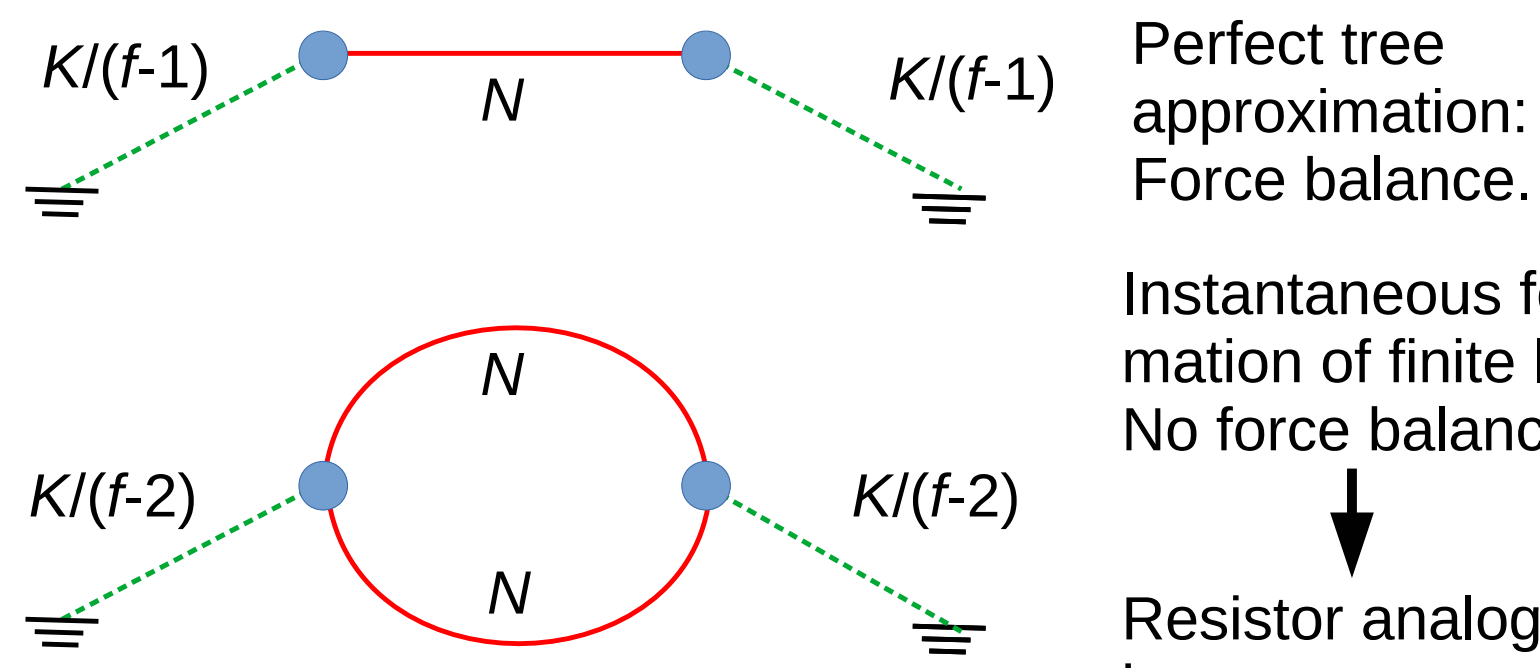

Instantaneous formation of finite loops: No force balance.

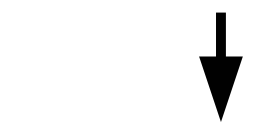

Resistor analogy is not exact 